\title{
5. FUNDAMENTAL WORK AT WASHINGTON
}

\author{
By F. P. ScOTT
}

\section{INTRODUCTION}

Each of the fundamental star catalogues prepared at the U.S. Naval Observatory may be regarded as a self-contained unit of work in which the positions derived for the objects therein are independent of any previous determination.

The basic procedures for our fundamental work have remained unchanged since the turn of the century. There have been, however, many modifications in the details associated with improvements in the instrument and with the use of new and better techniques for observing and computing. This discussion will deal mostly with the methods currently in use.

\section{InSTRUMental EguIPMENT}

Since Igoo our fundamental programmes have been conducted with the 6 in. and 9 in. transit circles. The new 7 in. transit circle, now being tested, is destined to replace the 9 in. instrument, which was retired from service I March 1945 .

The 7 in. transit circle was designed by Dr Watts of the U.S. Naval Observatory and has incorporated in it all the improvements which he developed for the 6 in. transit circle. It will be sufficient for this discussion to state that the 6 in. and 7 in. transit circles are equipped with photographic microscopes, motor-driven travelling threads and photographic recording for the right ascension and zenith distance micrometer screws.

\section{Fundamental Right Ascensions}

Two groups of stars, the clock stars and azimuth stars, play important parts in the determination of fundamental right ascensions. The clock stars comprise approximately 230 of the brighter $\mathrm{FK}_{3}$ stars well distributed in right ascension and declination between $+30^{\circ}$ and $-30^{\circ}$. The azimuth stars comprise about $\mathrm{I} 6$ stars within $10^{\circ}$ of the north pole. The best right ascensions available are provisionally adopted for the clock and azimuth stars at the beginning of a programme. The observations, reductions, and discussions have to be planned so as to provide all information required to:

(a) Correct the provisional system for systematic errors $\Delta \alpha_{\alpha}$ and $\Delta \alpha_{\delta}$.

(b) Correct the individual right ascensions of the provisional system.

(c) Correct the zero point of right ascension of the provisional system.

(d) Eliminate, as much as possible, all systematic errors of the instrument, the observers and the accessories.

A large portion of the discussions required to determine corrections $(a)$ and $(b)$ is based on clock corrections obtained as early as possible in the evening, around midnight and as late as possible in the morning.

A single clock correction usually consists of 6 to Io stars observed by a single observer within a period of 2 or $3 \mathrm{hr}$. During this period the observer obtains all the necessary instrumental constants to reduce his own observations. Corrections for the irregularities of the pivots are also applied in deriving the clock correction.

Until a few years ago the clock rate was determined by comparing clock corrections obtained a few days apart at about the same sidereal time and containing essentially the same stars. Corrections for personal equations were determined and applied for this and other purposes. This practice was abandoned, however, when by trial it was found that the Time Service rates for the clocks served our purposes much better. Currently no correction for personal equation is applied because the use of screens, reversing prisms, and impersonal micrometers has reduced it to a point where it is no longer detectable with the personal equation machine or by comparing the results of different observers.

Near the end of the programme the deviations of the clock corrections given by the 
individual stars from the observer's mean clock correction for a tour are tabulated according to declination for each clamp position of the instrument, provided:

(I) The clock stars involved in a clock correction have a spread in declination of $20^{\circ}$ or more.

(2) The mean declination of the clock stars employed is within $5^{\circ}$ of the equator. The resulting tabulation for the programme indicates the nature of the variation of the clock correction with declination and provides a means of reducing, when necessary, any clock correction to the equator $\left(\Delta \alpha_{\delta}\right)$. It also serves as a means of obtaining a preliminary value of the clamp difference to be used in later investigations.

The periodic errors introduced in the provisional clock corrections through the presence of similar errors in the provisional right ascensions are determined by means of comparisons of clock corrections taken during the same night. For this purpose the clock corrections for the nights on which two or more sets of clock stars were observed are reduced to the equator, if necessary, and with the adopted clock rates reduced to a common epoch, and compared. Each difference, morning minus evening clock correction, gives rise to an equation of the form:

$$
b\left(\sin \alpha_{E}-\sin \alpha_{M}\right)+a\left(\cos \alpha_{E}-\cos \alpha_{M}\right)+\ldots=\Delta T_{M}-\Delta T_{E}-\Delta S_{M}+\Delta S_{E}
$$

where $\alpha_{E}=$ right ascension of evening clock correction,

$\alpha_{M}=$ right ascension of morning clock correction,

$\Delta T_{M}=$ morning clock correction,

$\Delta T_{E}=$ evening clock correction,

$\Delta S=$ error in a clock correction due to errors in the individual places of the provisional system.

The quantities $\Delta S$ are usually quite small and are random enough in value so as to have but little effect on the first approximation to the coefficients of the periodic terms.

Ordinarily, during a programme, it is possible to set up 300 or more equations from the comparisons of suitable evening and morning clock corrections. The interval between the compared clock corrections usually ranges from 4 to II $\mathrm{hr}$. Before solving these equations it has been the practice to make all corrections required to the preliminary azimuth due to errors in the provisional right ascensions of the azimuth stars and due to the diurnal variation in the azimuth of the meridian marks.

The results of the solution for periodic terms are applied to the provisional right ascensions and all clock corrections are adjusted accordingly. The clock correction for each tour, now freed of periodic errors, is reduced to the time of transit of each clock star observed during the tour by means of the adopted clock rate. A comparison of the clock correction given by an individual star with the mean clock correction for the tour is, except for accidental errors, the correction to the provisional right ascension required by that star. For a single star, the average of all such comparisons is accepted as the correction to the provisional right ascension, proper attention to clamp difference being given when collecting the results. Usually no further revisions of the clock corrections are required.

The star places derived in the manner just described are used to compute the definitive clock corrections for the programme. The definitive clock correction for an observing tour, with the Time Service rates, are used to derive right ascensions for all objects observed during that tour.

The mean observed right ascensions, referred to the equinox of the provisional system, are formed by combining the observations made on each clamp, proper attention being given to clamp differences in making the combination. During the course of the programme care is exercised to distribute the observations as evenly as possible between the clamps and before and after midnight. The final step in our procedure for deriving fundamental right ascensions is the reduction of the observed right ascensions from the equinox of the provisional system to the equinox derived from a discussion of the Sun and planet observations. 


\section{Instrumental Constants in Right Ascension}

Ordinarily the level and azimuth constants are determined at the beginning and end of each $3 \mathrm{hr}$. tour of observing. The collimation of the $6 \mathrm{in}$. transit circle is usually determined around midnight. The azimuth of the instrument for a particular instant is obtained from observations of the meridian marks.

During the fundamental azimuth season, from September until the middle of May, the azimuths of the marks, for each period of about a month, are derived from an equal number of upper- and lower-culmination observations of each azimuth star. These observations are taken shortly after dusk and shortly before dawn. By using balanced observations of the circumpolars, the azimuths of the marks and the azimuth of the instrument are not affected by errors in the provisional right ascensions during the fundamental azimuth season.

During the summer months, when the nights are short, the azimuths of the marks for each month are derived from an equal number of upper culminations of certain stars and lower culminations of other stars. In order to minimize the effects of errors in the provisional star places, as many different stars as possible are observed.

Near the end of the programme, the observed right ascensions of the circumpolars, reduced with provisional clock corrections, are collected and a mean value formed for each star. This mean value is used for the revision of all azimuths of the marks for the purpose of correcting the non-fundamental summer azimuths and investigating the diurnal motion of the marks.

In addition to determinations of the level, azimuth and collimation, periodic investigations are made to determine the forms of the pivots and the errors of the right-ascension screw. Corrections for the form of the pivots are applied, but the errors of the micrometer screw are usually negligible.

\section{Fundamental Declinations}

The circle readings for all objects and the nadir are registered by means of four photographic microscopes. The zenith-distance micrometer readings are recorded on photographic tape. The nadir readings required for the reduction of the observations are obtained simultaneously with the level constant for right ascension. The barometric pressure is recorded at least once an hour and the temperature at about $20 \mathrm{~min}$. intervals.

The observed zenith distances are corrected for division errors, inclination, micrometerscrew errors, variation of latitude, reduction to meridian, and provisional refraction. The preliminary declinations are derived by applying the provisionally adopted latitude to the corrected zenith distances.

The preliminary declinations therefore contain errors of the adopted latitude, provisional refraction, and flexure as given by the collimators. Errors in these quantities propagate themselves over the sky in such similar fashion that it is impossible clearly to distinguish one from the other in the traditional circumpolar solution. The solution of the normal equations resulting from a comparison of above- and below-pole declinations is complicated by correlations between the unknowns and gives but a weak approximation to the quantities sought and must be further adjusted.

The observer usually does not have much to guide him in his decision as to the type of adjustment which will bring about the best reconciliation of the instrumental equator with the pole point given by the circumpolars. The decision is usually a matter of deciding on his relative confidence in the circumpolar solution and the flexure from the collimators. While it is true that a slight adjustment of both refraction and flexure might give the best results, it usually suffices to adjust only one of them on account of the correlations mentioned. In recent years the determinations of the flexure have been confined to the measurement of the coefficients of the simple sine and cosine flexures through the use of horizontal and vertical collimators. There has been a suspicion, but no concrete evidence, that the flexure from the horizontal collimators has been affected by systematic errors due to refraction in the line of sight between the telescope and collimators. It is because 
of this suspicion that an adjustment of the flexure, about to be described, was made for one of our star catalogues (Publ. U.S. Naval Obs. vol. XVI, part III).

This adjustment neglects $\Delta F$, the correction to the flexure, when solving the circumpolar observations for $K$ and $\Delta R, K$ being a constant which absorbs the constant errors in latitude and nadir, and $\Delta R$ being a correction to the adopted constant of refraction. The values of $K$ and $\Delta R$ so derived are in error due to neglecting $\Delta F$. If the Sun and planet observations are temporarily corrected for $K$ and $\Delta R$ they will yield a value of $\Delta \delta_{0}$, the correction to the equatorial declinations derived as already described, which will be of use in obtaining an adjustment in the values of $K, \Delta R$ and $\Delta F$. In an error-free declination system $\Delta \delta_{0}$ ought to be zero. If we assume $\Delta R$ given by the circumpolar solution to be correct, we may effect an adjustment in the values of $K$ and $\Delta F$ through the relations

$$
\begin{aligned}
& \Delta K+\Delta F \sin \left(90^{\circ}-\phi\right)=0, \\
& \Delta K+\Delta F \sin (-\phi)=\Delta \delta_{0} .
\end{aligned}
$$

This adjustment retains the pole point given by the circumpolars and distributes $\Delta \delta_{0}$ according to a sine law.

An adjustment of the type just described would be in error if the refractions to the north and south of the zenith were different or if the flexure did not follow a simple sine law. Trouble would also arise from a head-north minus head-south difference which persisted for some distance from the zenith.

The total correction required by all observed declinations for the case illustrated is computed from the formula

$$
\Delta \delta=\dot{K}+\Delta K+\Delta R \tan \zeta+\Delta F \sin \zeta
$$

The final declinations are formed by combining all observations on both clamps of the instrument, corrections for clamp differences being applied in the process so as to reduce all observations to the mean clamp. In this way any residual flexure which varies with the cosine of the zenith distance is eliminated.

\section{Sun and Planet Observations}

The Sun, Mercury, Venus, Ceres, Pallas, Juno and Vesta are observed for the purpose of determining corrections to the adopted equinox and instrumental equator. The observations of these objects are reduced in the same manner as star observations with additional corrections for parallax, phase, and motion being applied when necessary. As many observations of day-stars as possible are obtained for the determination of the night-minus-day correction required for the Sun, Mercury and Venus. The day-clock corrections for the day objects are obtained by extrapolating near-by night-clock corrections to the times of their respective transits by use of the Time Service clock rates.

The asteroids were added in I 949 with the hope that the night-time observations of such star-like objects would help to eliminate the uncertainties in the corrections to the equator and equinox introduced through ill-defined day terms and systematic differences between star and disk observations.

No conclusive test of the efficiency of the asteroids for this purpose is yet available. A solution of a poorly distributed series of observations of Ceres and Vesta taken with the 6 in. transit circle from I928 to I935 does indicate, however, that they will be of considerable value in the determination of the equator point. These observations indicated only a trivial correction, $+0{ }^{\prime \prime} 02 \pm 0 " 08$, to the equator adopted for the 6 in. transit circle work from I925 to I94I, thus confirming the corrections already applied.

\section{Star Lists Fundamentally Observed}

The U.S. Naval Observatory has always endeavoured to keep well recognized and generally accepted star lists under observation. For more than two decades the FK 3 stars have formed the framework around which our fundamental programmes have been 
built. The $\mathrm{FK}_{3}$ supplementary stars will soon be added to this list. In addition to such well-recognized star lists, many special lists of stars have found their way into our fundamental programmes. Among the special lists are the PZT lists for Washington, Richmond, Herstmonceux and Ottawa and a selection of $\mathrm{O}$ and $\mathrm{B}$ type stars from the list prepared by Dr A. Blaauw. A considerable portion of our observing time since r94I has been devoted to about 7200 reference stars between $+35^{\circ}$ declination and the North Pole.

For some time we have been deeply concerned with the sparsity of stars in the $\mathrm{FK}_{3}$, especially when endeavouring to compare our fundamental catalogues with a standard reference system or with similar catalogues produced elsewhere. To remedy this we have recently resorted to comparing through the GC because of its greater number of stars, a procedure which is not entirely satisfactory. The addition of the supplementary stars to the $\mathrm{FK}_{3}$ will greatly increase its value as a catalogue of comparison.

\title{
6. ON THE FUNDAMENTAL ASTROMETRIC WORK OF THE CAPE OBSERVATORY
}

\author{
By R. H. STOY
}

At the Cape we are engaged on two major astrometric projects. The first of these, the Cape Photographic Catalogue for I950.0, includes 65,000 stars from $-30^{\circ}$ to $-40^{\circ}$ and from $-52^{\circ}$ to the South Pole. It is a differential rather than a fundamental catalogue, but it should provide the material from which a really satisfactory selection of faint stars for a fundamental catalogue may be made. The astrometric observations are complete and it is hoped that the work as a whole will be finished by 1960 .

The second astrometric project is the series of fundamental observations with the Gill Reversible Transit Circle which is to form the basis of the Second Cape Catalogue for I950.0. The working list now contains approximately 7000 stars and includes the usual Cape clock and azimuth stars, all $\mathrm{FK}_{3}$ stars south of $-20^{\circ}$, all GC stars brighter than $8^{\mathrm{m}} \cdot 5$ between $-30^{\circ}$ and $-52^{\circ}$ and between $-80^{\circ}$ and the South Pole, a selection of stars south of $-80^{\circ}$ to serve as standards for the reduction of the photographic zone observations, and a number of extra stars added recently. These extra stars include 396 stars between $-29^{\circ} 30^{\prime}$ and $-40^{\circ}$ which, together with the stars already in the working list, are intended to provide a sufficient number of evenly distributed stars for the reduction of photographic plates to be taken with the Yale Observatory Camera in Australia. They also include 65 stars south of $-30^{\circ}$ taken from FKSZ list of 93I faint fundamental stars. The other 22I stars of this list south of $-30^{\circ}$ were already included in the working list or have been observed with the Gill Reversible Transit Circle within the last ten years. It has not been considered necessary to add to the present working list any of the 845 stars from the recently published $\mathrm{FK}_{3}$ Supplementary List, as all these stars were either already included or have been observed at the Cape relatively recently. Our intention is to observe, as far as it may be possible, each star eight times, four times in each position of the instrument. Observations of the Sun, Mercury, Venus and a number of bright stars are attempted five days a week. The Moon, Ceres, Juno, Pallas and Vesta are observed whenever possible and other planets twice a week. The observations for this catalogue were begun at the end of I95I and it is hoped that they will be completed before the end of I957. Those for the polar section are already completed and are to be reduced together with the corresponding photographic observations before the remainder of the catalogue.

The observations made with the Gill Reversible Transit Circle have entered so fully into the constitution of the southern portions of the various fundamental catalogues that it may be thought that future work with it can do little to improve the present fundamental systems. This rather pessimistic view may be partially true and undoubtedly some of the present systematic errors can be discovered and eliminated more easily by 